\section{Biology of} cancer

Cancer: A Problem of Developmental Biology. By G. B. Pierce, R. Shikes and L. M. Fink. Pp. 242. (Prentice-Hall: Englewood Cliffs, New Jersey, 1978.) $£ 11.65$.

REAIDERS of that neglected masterpiece, The Crock of Gold (J. Stephens, Macmillan, 1928), will remember the philosopher's comment (to his wife) on the porridge - "Perfection is finality, finality is death. Nothing is perfect. There are lumps in it." Like the porridge, Cancer: $A$ Problem of Developmental Biology has some indigestible lumps in it, but for all that it is a useful book for those for whom it is intended (graduate students). Don't be put off by the modest aims outlined (one hopes by the publisher) on the book cover:

"The unifying theme of this modern approach to the study of tumors is development and neoplasia, treating carcinogenesis as a model of differentiation rather than as a neoplasmic entity alone. Coverage includes chapters devoted to the early development of neoplasms, tumors as caricatures of tissue renewal, structure and biochemistry of neoplasms, origin of neoplastic stem cells, carcinogenesis, genetics and neoplasia, controls, immunity and neoplasms, metastasis, and caner therapy.

"An appendix concerning various types of tumours also is included. Among the features: Emphasizes the importance of cellular interaction in tumors, showing their cellular heterogeneity and control mechanisms; Presents an excellent introduction to the clinical phenomenology of cancer, placing more specialized aspects in a useful perspective; Organized into a logical, unified, and complete volume that includes the results of most recent research; Provides a complete bibliography of sources of more specialized information; Includes a chapter on therapy and some brief descriptions on the commonly studied tumors."

It does discuss many of these aspects but not in exhaustive detail, in its 199 pages of text. It does not compare in depth with Foulds' Neoplastic Development (Academic, 1969) which is still the best general introduction for the specialist to the biology of cancer. Cancer: $A$ Problem of Developmental Biology is designed as an introduction to the field and in this I think that it achieves its purpose. For the student of biology with a general interest in cancer or a neophyte about to plunge into some highly specialised branch of cancer research, Pierce et al. will provide a useful reminder that there is more to cancer than molecules and also remind its readers that many of the findings of earlier workers are still relevant.

The faults of the book are superficial irritants rather than major defects, but nevertheless they are important, particularly in a book intended for students. The emphasis on the interests of the authors is understandable and not excessive, but the style sometimes grates. There has to be a better way of saying "respond negatively in terms of growth rate", particularly if one wants students to learn conciseness of expression.

A more serious criticism is concerned with minor but important inaccuracies and omissions. It is easy for a reviewer to carp over trifles, particularly when the authors are trying to compress a mass of information into a small space, but it is important not to mislead. A few examples will illustrate the point. The given definition of malignant tumours states that the malignant cells "are intrinsically dangerous because they are rapidly growing and quickly cause cachexia or they invade and metastasize. The intrinsically dangerous cells of malignant tumours invariably destroy their host unless they are either extirpated or killed". Although this is often so, the variation in growth rate of tumours is well known as is the whole concept of latency in tumours and of carcinoma in situ, which are in fact discussed elsewhere. The appropriate qualifying clauses would clarify the situation - important for those using the index to look for a definition of malignancy.

On the experimental side the authors suggest that transformation in vitro should produce a spectrum of cell lines, some

\section{High performance liquid chromatography}

High Performance Liquid Chromatography. Edited by J. H. Knox. Pp. 203. (Edinburgh University Press: Edinburgh, 1978.) £5.

THIs book is based on the material of a course on high performance liquid chromatography (HPLC) given in 1977 at the Wolfson Liquid Chromatography Unit, of which Professor Knox is the director. It represents a commendable attempt to put both the theory and practice of the subject on a firm physicochemical foundation, which the student can use to attack his own problems. The main theory of liquid chromatography is compressed into the relatively short chapter 2, followed by successive chapters on the familiar subdivision of chromatographic methods according to retention mechanism, adsorption, liquid-liquid partition, and ion-exchange (including ion-pair) and exclusion chromatography. These topics are very fully treated in the space available, and each chapter includes a table of the appropriate column packing materials commercially available.

Unfortunately the theoretical treatment tumour producing, some not-“an idea that has escaped attention", although they quote elsewhere a paper describing this very phenomenon. Sadly (as the paper is one of mine) it is given as a reference to work it does not describe.

In the general discussion on spontaneous transformation in vitro the authors fail to mention that this is predominantly a feature of some rodent cells and does not occur in species in which cells have been shown to have a finite lifespan as in Hayflick's experiments with human cells. In the section on genetics and neoplasia a statement is made that "recently, viral particles, similar to those of mouse mammary tumour virus, have been discovered in the milk of a high percentage of Parsi women" (Moore et al., 1971), with the implication that these particles are true viruses and associated with a high incidence of breast cancer in these women. Many would no longer accept these findings. Other examples can be cited.

In spite of the criticisms this could be a useful book but it would be greatly improved by a more critical assessment of statements, definitions and references.

L. M. Franks

L. M. Franks is in the Department of Clarellu Pathology at the Imperial Cancer Research Fnnd Laboratories, London, UK.

of chapter 2 is rendered unnecessarily confusing by an unfamiliar notation in which $\mathrm{V}_{\mathrm{m}}$, the volume of eluent within the column, is taken to include both the liquid in the interstitial spaces between the packing particles and the liquid within the stationary phase pores. Although the pore volume cancels out in the definition of phase capacity ratio $k$ (equation $2.2, \mathrm{p} 5$ ), it can only be valid as a mean value in the definition of linear flow velocity $\mu$ (equation 2.3). It leads to a new definition of zone capacity ratio $\mathrm{k}$ " on p7, which is almost immediately abandoned on $\mathrm{p} 8$, leading to the more usual relationships of equations (2.122.17). The concept is again introduced in the treatment of the column resistance factor on pp9-10. This notation is all the more surprising, as the authors found it unnecessary in their excellent theoretical treatment in an earlier book.

Perhaps the most unfortunate result appears however in chapter 7 on exclusion chromatography, where an essentially simple concept is confused by the introduction of three definitions for the distribution ratio between the phases (p69). This chapter also illustrates the preoccupation of practitioners of HPLC with the exclusion chromatography of synthetic polymers, usually uncharged and soluble only in organic solvents. Although there is a brief reference to the 